\title{
Conséquences des accidents radiologiques sur la santé mentale
}

\author{
Ph. PIRARD*, J. BRENOT*, P. VERGER*
}

(Manuscrit reçu le 12 janvier 1998, révisé le 25 juillet 1998, accepté le 25 aout 1998)

RÉSUMÉ Les catastrophes technologiques entraînent des troubles psychologiques plus ou moins marqués et durables au sein des populations concernées. Cet article présente une revue des études épidémiologiques effectuées après les accidents de Three Mile Island, de Goiânia et de Tchernobyl pour en évaluer les conséquences psychologiques. Elle montre, dans des contextes culturels et accidentels très différents, une augmentation significative et durable des symptômes psychologiques dans différents groupes de population exposée, en faveur d'une détresse psychologique réelle. II y a moins de résultats en faveur de l'existence d'une augmentation des syndromes psychiatriques, mais ces effets ont été beaucoup moins étudiés. Des groupes où les effets semblent avoir été plus marqués ont été identifiés tels les mères de jeunes enfants, les personnes relogées, les personnes ne pouvant bénéficier d'un soutien social de qualité ou en situation de précarité économique. La détresse psychologique observée peut favoriser le développement de troubles psychiatriques et donner lieu à des changements de comportement à l'égard de la santé. Des recherches restent nécessaires pour mieux préciser la nature des troubles rencontrés et leurs déterminants. Elles devraient s'appuyer sur la construction d'outils mieux adaptés notamment à l'évaluation des circonstances individuelles d'exposition et au diagnostic des effets.

\section{ABSTRACT The aftermath of nuclear accidents on mental health}

Technological disasters bring about psychological effects in exposed populations of various durability and intensity. This article reviews the epidemiological studies which assess psychological and psychiatric consequences of the Three Mile Island, Goüania and Chernobyl accidents. It shows, in different accidental and cultural contexts, a statistically significant and durable increase of psychological symptoms in various exposed population groups, which points out an actual psychological distress. Diagnosed psychiatric effects are less constant, but much less studied. Most affected groups are mothers of young children, relocated persons, persons with less social support or in financial trouble. The psychological distress can further psychiatric disorders and give rise to behavioural changes towards health. More research is necessary to delineate the nature and the determinants of the observed symptoms and disorders. It implies to design better tools for the assessment of individual exposure and the diagnosis of mental health effects.

\footnotetext{
* Institut de Protection et de Sûreté Nucléaire, Département de Protection de la santé de l'Homme et de Dosimétrie,
} Service d'Evaluation et de Gestion des Risques, B.P. $n^{\circ} 6$ - 92265 Fontenay aux Roses, Cedex 3, France. 


\section{Introduction}

Les accidents radiologiques graves entrainent des effets sanitaires au sein des populations qui sont dus non seulement à l'exposition aux rayonnements mais aussi aux désorganisations environnementales, sociales et économiques qui s'ensuivent. Aux maladies somatiques aiguës ou chroniques, s'ajoutent des troubles psychologiques plus ou moins marqués et durables. Les pouvoirs publics ont à gérer les conséquences de ces accidents avec en particulier l'information de la population et la mise en place de mesures de protection adaptées aux besoins des populations touchées. Ceci n'est possible que si des évaluations bien conduites précisent la nature des problèmes qui se posent et les quantifient. Il en est ainsi des problèmes de santé publique.

Cet article présente une revue des conséquences sur la santé mentale de trois accidents radiologiques survenus dans les vingt dernières années : Three Mile Island, Goiânia et Tchernobyl. Les effets psychologiques de ces accidents sont nombreux et recouvrent notamment la souffrance psychique, les modifications des perceptions des risques et les modifications des comportements individuels et sociaux. Cette revue est volontairement centrée sur la souffrance psychique. Celle-ci peut se manifester sous la simple forme de la perception d'une atteinte du bien-être psychique. Elle peut prendre des formes intenses et handicapantes dans la vie quotidienne et parfois s'accompagner de manifestations cliniques bien caractérisées et définies comme pathologiques. Ces différents aspects recouvrent ainsi, dans le domaine du mental, les atteintes à la santé telle qu'elle est définie par l'OMS : un état de bien-être mental, physique et social (Kovess, 1986). Dans cet article, il s'agit de voir s'il est possible de répondre objectivement aux assertions faites par les médias ou les responsables qui font état de l'importance du problème de santé publique posé par les atteintes psychologiques des populations exposées.

Les types d'effets étudiés et les outils d'évaluation utilisés sont d'abord présentés. Une revue des études épidémiologiques traitant de santé mentale à propos des trois accidents est ensuite effectuée. Enfin, différentes voies de recherche sont proposées pour améliorer les connaissances sur l'impact psychologique des catastrophes radiologiques. Notons que ces voies de recherche sont en grande partie applicables aux autres catastrophes environnementales d'origine naturelle ou anthropique.

\section{Aspects méthodologiques généraux}

La distinction entre court terme et long terme après l'accident est utile, car d'une phase à l'autre, les effets peuvent être de nature différente ou voir leur niveaux évoluer, les groupes d'individus à étudier peuvent ne pas être les mêmes et la démarche du chercheur de spontanée peut devenir planifiée. Les études sont considérées comme à court terme quand l'évaluation a été faite dans l'année suivant l'accident. 


\subsection{Effets étudiés}

Les manifestations de l'impact psychologique des catastrophes environnementales sont très variées et généralement reliées à la notion de stress. Ce terme, rarement défini, rend compte d'une situation continue de désadaptation biologique, psychique et sociale qui exige de l'organisme, du système nerveux et de l'espace psychique, un état d'alerte, une surtension et une dépense d'énergie permanents (Hériard-Dubreuil, 1994). Cette réaction physiologique inévitable est étroitement liée à l'appréciation par l'individu de la situation où il se trouve. Elle peut se traduire de différentes façons, par exemple par l'augmentation de la sécrétion de certaines hormones ou par une modification des capacités de concentration. Son intensité et sa durée peuvent donner lieu à une atteinte prolongée du bien-être. D'autres manifestations sont l'apparition de symptômes d'anxiété, de dépression ou psychosomatiques et des modifications du comportement. Ces dernières se traduisent par l'accroissement de la consommation de médicaments (anxiolytiques, antidépresseurs) ou de produits toxiques (alcool, tabac...), de consultations médicales ou psychologiques, voire même de la fréquence des comportements déviants (crimes et délits...). L'atteinte prolongée du bien-être peut favoriser la survenue de pathologies post-traumatiques plus prononcées : des troubles anxio-névrotiques (anxiété généralisée, attaques de panique...), des troubles dépressifs, la décompensation d'affections psychiatriques préexistantes ou la survenue de maladies psychosomatiques. L'exposition à des facteurs de stress de forte intensité, menaçant par exemple de façon soudaine et imminente l'intégrité de la personne, peut entrainer un état de stress post-traumatique. Ce syndrome associe des souvenirs très précis, répétitifs, envahissants et irrépressibles de l'événement (pensées intrusives) et des symptômes psychologiques et somatiques liés à des conduites d'évitement et une hyperactivité neurovégétative. Ce syndrome, qui peut être très handicapant et persister plusieurs années, a été peu recherché à la suite des accidents radiologiques.

La définition de ces atteintes ne fait pas toujours consensus. Néanmoins des efforts de standardisation ont été entrepris par l'American Psychiatric Association dans son guide « Diagnostic and Statistical Manual of Mental Disorders, III ${ }^{e}$ révision » (DSM IIIR) et par l'Organisation Mondiale de la Santé dans sa $\mathrm{X}^{\mathrm{e}}$ révision de la Classification Internationale des Maladies (CIM-10) (ICD 10).

\subsection{Méthodes et outils d'évaluation}

Le recueil de données se fait selon deux modes principaux, directement en interrogeant les individus ou indirectement en observant leurs comportements.

À l'échelle individuelle, l'interrogation peut prendre la forme d'entretiens individuels en profondeur par un psychologue ou un médecin en utilisant les guides d'entretiens structurés standardisés construits à partir du DSM IIIIR ou de la CIM10. Certains comme le « Schedule for Affective Disorders and Schyzophrenia-Lifetime Version 
(SADS-L) » (Endicott et al., 1978) ou le Munich Diagnostic Check-List (Hiller et al., 1990) couvrent aussi bien les troubles anxieux et de l'humeur que les troubles psychotiques ou d'origine organique. L'interrogation réclame du temps et des moyens, aussi se fait elle le plus souvent sur de petits échantillons.

Les instruments de mesure les plus utilisés sont les échelles de symptômes (checklist) qui permettent d'établir des scores relatifs à différents types de troubles (anxiété, dépression, troubles psychosomatiques...). Les réponses sont recueillies par entretien en face à face ou téléphonique ou par auto-questionnaire. L'instrument le plus fréquemment utilisé après l'accident de Three Mile Island est le « Symptom Check-List 90R » (SCL90R) (Derogatis, 1977). Le « Behavior Schedule Questionnaire (BSQ) » (Richman et al., 1971) a été utilisé pour évaluer le comportement des jeunes enfants après l'accident de TMI. Pour Tchernobyl, il s'agit du General Health Questionnaire (GHQ 12 ou 28 items) (Goldberg, 1978). Ce dernier présente l'avantage d'avoir été traduit dans de nombreuses langues et validé en population générale. Il existe d'autres échelles pour détecter l'existence d'un état de stress post-traumatique comme l'Impact of Event Scale (Horowitz et al., 1979).

À l'échelle collective, l'atteinte au bien-être ou à la santé mentale est appréciée par des indicateurs indirects tels que les niveaux de vente d'alcool, de médicaments anxiolytiques, le nombre de consultations médicales ou même les comportements sociaux déviants (actes de criminalité, délit...).

\subsection{Autres facteurs étudiés}

Certains facteurs peuvent modifier la gravité ou la nature des effets observés. Ils sont le plus souvent démographiques (sexe, âge...). Dans certaines études, des facteurs individuels d'ordre psychologique ont été étudiés comme, par exemple, les perceptions de l'environnement et des dangers liés à l'accident, les attitudes adoptées face au futur et les façons de s'adapter aux risques (coping strategies). Des facteurs sociologiques sont parfois considérés, comme les stratégies de gestion communautaire ou la nature et la qualité de l'environnement social (amis, famille, confidents...).

\section{L'accident de Three Mile Island}

Le 28 mars 1979, un accident survenait sur la centrale nucléaire de Three Mile Island en Pennsylvanie, USA. Cet accident, aux conséquences matérielles très lourdes, a entraîné une exposition de la population aux rayonnements très inférieure à celle due à la radioactivité naturelle (Fabrikant, 1981). Mais les réactions de la population ont été importantes, probablement en raison de la gestion de l'information par les autorités sur l'état de la centrale et les recommandations de mesures de protection (Bartlett et al., 1983) : 200000 personnes environ se sont enfuies de la région dans les jours suivant l'accident. Plus tard, différents facteurs ont contribué à faire durer l'inquiétude. 
La survenue de l'accident, pourtant déclaré comme très improbable, a été perçue par le public comme une faillite des scientifiques, des experts et des autorités décrédibilisées par leur gestion de la crise. Dès lors, les informations officielles sur les doses reçues et les risques ont été contestées. De plus, les lâchés de gaz radioactifs successifs lors de la réhabilitation du site puis le redémarrage de la centrale en 1985 , ont été abondamment médiatisés, constituant autant de rappels de l'accident.

Dès les premiers jours, dans l'urgence, des enquêtes étaient réalisées dans la population (Dohrenwend, 1981). Dans les premiers mois, le gouvernement fédéral, le Nuclear Research Council et le département de la santé de Pennsylvanie demandaient la mise en place d'études pour évaluer les conséquences économiques, sociales, sanitaires et psychologiques de l'accident (Kemeny et al., 1979). L'évaluation de l'impact sur la santé mentale était un objectif important de ces études.

\subsection{Effets sur la santé mentale à court terme}

\subsubsection{Populations étudiées}

Les études ont été effectuées au sein de la population générale (Houts et al., 1984; Dohrenwend et al., 1981, 1983) et dans des groupes particuliers (Bromet et al., 1982; Dohrenwend et al., 1981, 1983; Fabrikant, 1983) :

- les femmes enceintes et les mères de jeunes enfants, cibles principales des recommandations du gouverneur;

- les patients des centres de soins mentaux a priori fragilisés;

- les employés de la centrale;

- les collégiens, autres cibles des recommandations du gouverneur.

Les personnes résidant à moins de 8,16 ou 32 kilomètres de la centrale ont été considérées comme exposées. Les enquêtes spontanées ne comportaient pas, pour la plupart, de groupe non exposé. Cependant certains auteurs ont cherché à comparer les groupes exposés à des groupes situés à plus de $60 \mathrm{~km}$ de la centrale ou proches d'autres centrales nucléaires (Bromet et al., 1982).

\subsubsection{Résultats}

Les mères et les patients des centres de soins mentaux ont perçu en majorité l'accident comme dangereux pour la santé. La proportion est plus importante pour les exposés que pour les non exposés ( $>60 \%$ contre $<55 \%$, pas de test de significativité). Par contre, à peine $25 \%$ des employés de la centrale accidentée ont déclaré que l'accident leur paraissait dangereux pour leur santé et $33 \%$ pour les employés d'autres centrales (Bromet et al., 1982). 
Dans la population générale, $52 \%$ des individus ont évacué leur domicile dans les $16 \mathrm{~km}$ autour de la centrale et $62 \%$ dans les $8 \mathrm{~km}$ (Dohrenwend et al., 1981). Toutes les mères dans les $8 \mathrm{~km}$ ont évacué leur domicile et $75 \%$ d'entre elles dans les $16 \mathrm{~km}$. Certains auteurs ont observé dans les populations exposées, une augmentation significative de la consommation de somnifères et d'anxiolytiques durant la période de crise (Houts et al., 1984; Mac Leod et al., 1982). Une nette augmentation de la consommation d'alcool dans un rayon de 5 à $10 \mathrm{~km}$ autour de la centrale dans les jours qui ont suivi l'accident a été mise en évidence (Mileti et al., 1984). Houts et al. n'ont pas observé de modification de la consommation de soins médicaux dans l'année qui a suivi l'accident (Houts et al., 1984).

En ce qui concerne les échelles d'anxiété et de dépression, les scores les plus élevés étaient observés au moment de l'accident. Ils ont diminué dans les 6 mois suivants (Dohrenwend et al., 1981 ; Houts et al., 1984).

Dans l'année qui a suivi l'accident, l'incidence d'épisodes anxieux et dépressifs sévères chez les mères exposées était significativement plus élevée que celle relevée dans un groupe non exposé. Une multiplication par 4 de la prévalence de ces troubles était observée en avril 1979 chez les mères exposées. Par contre, aucune différence significative ni pic n'étaient constatés chez les patients de centres de soins mentaux. L'incidence d'épisodes anxieux et dépressifs chez les employés de la centrale était significativement plus importante que celle de leur groupe comparatif, mais avec des niveaux bien plus bas que chez les mères ou les patients de centres de soins mentaux (Tab. I) (Bromet et al., 1982).

\section{TABLEAU I}

Troubles psychiatriques dans les populations exposées de TMI et celles résidant autour de la centrale de Beaver (d'après Bromet et al., 1982)*. Mères de jeunes enfants.

Psychiatric disorders in relation to the TMI accident (Bromet et al., 1982). Mothers of young children.

\begin{tabular}{|c|c|c|c|c|}
\hline (t) & \multicolumn{4}{|c|}{ Troubles olpressitis ou madeux } \\
\hline Forighe & $\begin{array}{l}\text { nombre Xw1 } \\
\text { (BID wijes) }\end{array}$ & 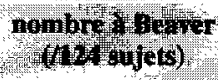 & $\begin{array}{l}\text { Risque relatil } \\
\text { TWUBbeaver }\end{array}$ & $1 \mathrm{C} * \mathrm{a} .55 \%$ \\
\hline un an avant TMI & 84 & 31 & 1,08 & $0,76 \cdot 1,53$ \\
\hline $\begin{array}{l}\text { nouveaux cas } \\
\text { un an après TMI }\end{array}$ & 25 & 3 & 3,4 & $1,16-9,98$ \\
\hline \multicolumn{5}{|c|}{ 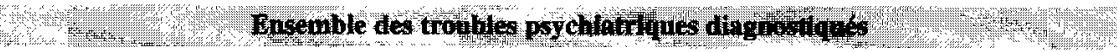 } \\
\hline un an avant TMI & 93 & 44 & 1,23 & $0,87-1,75$ \\
\hline $\begin{array}{l}\text { nouveaux cas } \\
\text { un an après TMI }\end{array}$ & 26 & 3 & $\mathbf{3 , 7 2}$ & $1,29-10,76$ \\
\hline
\end{tabular}

* Questionnaire utilisé : SADS-L (Schedule for Affective Disorders and Schyzophrenia-Lifetime). ** IC : Intervalle de Confiance. 
Habiter à proximité de la centrale au moment de l'accident pour l'ensemble des groupes étudiés ou avoir des antécédents de pathologies psychiatriques pour les mères et les employés constituaient des facteurs aggravants (Dohrenwend et al., 1981; Bromet et al., 1982). Bénéficier d'un bon soutien social était un facteur protecteur (Bromet et al., 1982).

\subsubsection{Discussion}

Les études doivent être interprétées avec prudence. En plus de l'absence fréquente de groupe non exposé, les outils de mesure utilisés n'avaient pas été validés et n'étaient pas identiques d'une étude à l'autre. Ces études ont montré néanmoins l'existence d'effets psychologiques immédiats dans la population et la sensibilité particulière des mères de jeunes enfants, chez lesquelles des troubles sévères ont été observés. Pour l'ensemble des groupes, les effets ont diminué dans l'année suivant l'accident tout en restant significativement plus élevés pour les mères et les employés exposés comparativement aux groupes témoins (Tab. I et II).

\section{TABLEAU II}

Troubles psychiatriques dans les populations exposées de TMI et celles résidant autour de la centrale de Beaver (d'après Bromet et al., 1982)*. Personnel des centrales.

Psychiatric disorders in relation to the TMI accident (Bromet et al, 1982). Nuclear plant workers.

\begin{tabular}{|c|c|c|c|c|}
\hline \multicolumn{5}{|c|}{ Toobles dépressifs ou gnxieux } \\
\hline Pertode & nombre o TMI & $\begin{array}{l}\text { nombre a Beaver } \\
(\text { (124 sujets })\end{array}$ & $\begin{array}{l}\text { Ropoe rewir } \\
\text { MMriearer }\end{array}$ & $1 C^{4}-95$ \\
\hline un an avant TMI & 41 & 18 & 1,75 & $1,08 \cdot 2,86$ \\
\hline $\begin{array}{l}\text { nouveaux cas } \\
\text { un an après TMI }\end{array}$ & 11 & 3 & 3,24 & $1,00-10,44$ \\
\hline no & \multicolumn{4}{|c|}{ Ensemble des troubles psychintriques diagnostiqués } \\
\hline un an avant TMI & 61 & 34 & 1,38 & $0,98-1,94$ \\
\hline $\begin{array}{l}\text { nouveaux cas } \\
\text { un an après TMI }\end{array}$ & 7 & 3 & 2,1 & $0,58-7,63$ \\
\hline
\end{tabular}

* Questionnaire utilisé : SADS-L (Schedule for Affective Disorders and Schyzophrenia-Lifetime).

*** IC : Intervalle de Confiance.

\subsection{Effets sur la santé mentale à long terme}

Des évaluations répétées ont été effectuées pendant plusieurs années dans les types de populations précédemment étudiées (Baum et al., 1993; Bromet et al., 1990; Dew et al., 1987 et 1993). Toutes les études ont inclus des groupes non exposés choisis dans 
les périmètres équivalents d'une autre centrale nucléaire de l'est des États-Unis. En plus des troubles précédents, les troubles du comportement chez les jeunes enfants d'âge préscolaire ont été étudiés (Cornely et al., 1986). Les auto-questionnaires utilisés étaient validés et identiques ( $c f .2 .2$ ). Les auteurs ont aussi étudié des facteurs susceptibles d'être associés à une plus grande sensibilité à l'impact psychologique de l'accident (Fleming et al., 1982; Dew et al., 1987). Des mesures biologiques ont été effectuées, notamment le dosage urinaire de la norépinéphrine, ainsi que des tests psychotechniques de performance (Baum et al., 1993; Gatchel et al., 1985).

\subsubsection{Résultats}

Dans la même étude, Gatchel (1985) et Baum (1993) ont suivi des échantillons issus de la population générale jusqu'à dix ans après l'accident. Pour les six premières années, les diverses mesures ont montré un état de stress des populations exposées significativement supérieur à celui des populations non exposées (Tab. II). Différentes manifestations physiologiques du stress ont été relevées : accroissement du niveau de norépinéphrine dans les urines et diminution des performances aux tests psychotechniques. Des scores de symptômes anxieux, dépressifs et somatiques (SCL90R) significativement plus élevés ont été constatés. De même, une partie de la population a présenté des scores de stress post-traumatique plus élevés (Impact of Event Scale). Au-delà de six ans, les différences entre exposés et non exposés n'étaient plus significatives (Tab. III).

\section{TABLEAU III}

Effets au long terme de l'accident de TMI en population générale - études transversales répétées (d'après Baum et al., 1993).

Long term effects of the TMI accident in general population - different time periods (Baum et al., 1993).

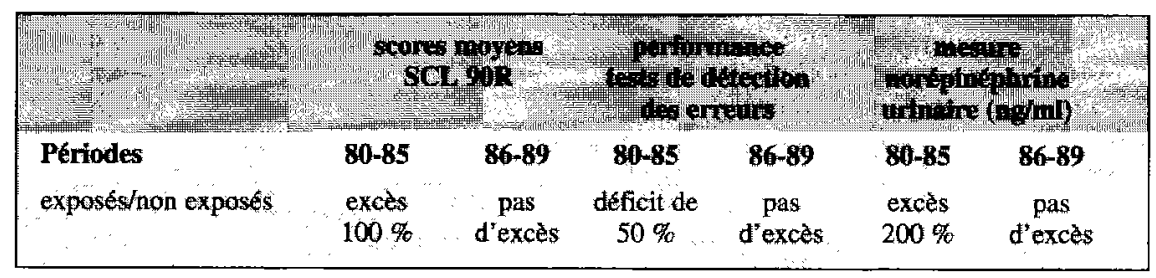

Les employés de la centrale ont été suivis jusqu' au $42^{\mathfrak{e}}$ mois après l'accident. Après la première année, il n'y avait plus de différence significative entre exposés et non exposés (Bromet et al., 1990). Concernant les mères exposées, l'incidence des épisodes psychiatriques d'ordre anxieux et dépressif n'était plus supérieure à celle des mères non exposées dès la deuxième année après l'accident. Les scores de détresse 
psychologique sont restés les mêmes au cours des années suivantes parmi les mères exposées. Une augmentation des scores de symptômes a été observée au trentième mois chez les mères non exposées. Elle a été attribuée par les auteurs à la survenue au même moment d'une crise économique dans la région du groupe en question (Bromet $e$ t al., 1990). Une remontée des scores de symptômes a été mise en évidence chez les mères exposées lors du redémarrage de la centrale et lors du dixième anniversaire de l'accident (Dew et al., 1987 et 1993). Certains facteurs étaient associés à des effets plus élevés, tels que le fait d'habiter à proximité de la centrale, de s'être enfui au moment de l'accident, d'avoir un soutien social précaire ou d'avoir des antécédents d'épisodes d'anxiété et de dépression (Fleming et al., 1982; Dew et al., 1993).

Chez les enfants, les comparaisons de la prévalence des troubles du comportement effectuées deux ans et demi après l'accident entre les groupes exposés et non exposés n'ont pas montré de différence. De plus, la perception du danger lié à l'accident chez les mères du groupe exposé n'est pas corrélée aux troubles du comportement observés chez leurs enfants (Cornely et al., 1986).

Dans tous les groupes étudiés, les individus se répartissent en deux grandes catégories (Baum et al., 1993; Dew et al., 1993). La première est constituée d'individus chez lesquels les effets psychologiques et psychiatriques sont importants et réactivés par des événements rappelant l'accident; elle représente environ le quart des individus sélectionnés dans les échantillons étudiés dans la cohorte suivie par Bromet et Dew. La seconde regroupe les trois-quarts des individus restants pour lesquels l'impact psychologique semble peu important et les résultats des évaluations sont stables.

\section{L'accident de Goiâna}

La récupération par des ferrailleurs d'un appareil de téléthérapie abandonné et la dispersion de la source de césium 137 qu'il contenait ont abouti, en septembre 1987, à de graves irradiations de personnes (4 décès par syndrome aigu d'irradiation) et à la contamination d'un quartier de la ville de Goiânia (Brésil). Environ 110000 personnes ont demandé un examen anthropogammamétrique et plusieurs milliers de personnes non contaminées se sont plaintes de symptômes divers (diarrhées, nausées, éruptions, phlyctènes). Enfin, l'accident a eu des répercussions importantes sur l'économie de l'État de Goias : baisse des activités touristiques et agricoles, chute des prix des biens immobiliers. La population de Goiânia a fait l'objet d'une véritable discrimination de la part du reste du Brésil (Petterson, 1988).

Collins et de Carvalho, (Collins et de Carvalho, 1993) ont cherché à quantifier, trois ans et demi après l'accident, le niveau de stress dans trois groupes de population tirés au sort : 25 personnes de Goiânia fortement irradiées lors de l'accident (doses comprises entre 0,5 et $1 \mathrm{~Sv}$ ) mais sans conséquence somatique observée, 25 personnes résidant dans un rayon de 1,5 à $2 \mathrm{~km}$ du lieu de stockage des déchets 
radioactifs récupérés à Goiânia (Abadia), 23 personnes sélectionnées dans un quartier de Goiânia non touché par l'accident. Le stress a été mesuré par des tests psychotechniques de performance, des dosages hormonaux urinaires reflétant l'activité du système nerveux sympathique (acide vanyl mandélique), la mesure de la tension artérielle et par différents auto-questionnaires dont une échelle de mesure de la peur et le « Cornell Medical Index » (Brodman et al., 1974). Ce dernier est composé de 10 échelles qui mesurent notamment la détresse, l'inadaptation et divers symptômes psychosomatiques. D'autres facteurs susceptibles d'influencer les résultats ont été pris en compte : la peur, le degré de perception des risques, l'état de santé général (Overall Health Inventory) ainsi que le niveau socio-économique.

\section{TABLEAU IV}

Comparaison de résultats de tests par autoquestionnaire, de mesures de la tension artérielle et de concentration d'acide vanyl mandélique dans les urines de personnes exposées ou menacées par la présence de ${ }^{137}$ Cs lors de l'accident de Goiânia en 1987 (d'après Collins et de Carvalho, 1993).

Comparison of self-report, cardiovascular and neuroendocrine indices of persons threatened by or exposed to ${ }^{137}$ Cs from the 1987 , Goïania, Brazil, nuclear accident

(Collins and de Carvalho, 1993).

\begin{tabular}{|c|c|c|c|}
\hline 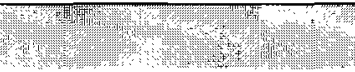 & Abryta & Gollinis & Tapoin \\
\hline Effectifs & 23 & 23 & 21 \\
\hline $\begin{array}{l}\text { Symptômes de détresse } \\
\text { et d'inadaptation (GHQ 28) }\end{array}$ & $7,8^{* * * *} \pm 0,8$ & $6,8 * * * \pm 0,7$ & $3,8 \pm 0,5$ \\
\hline $\begin{array}{l}\text { Symptômes somatiques } \\
\text { (GHQ28) }\end{array}$ & $30,8^{*} \pm 3,5$ & $31,4^{*} \pm 3,4$ & $20,6 \pm 2,5$ \\
\hline Perception du danger & $0,7 \pm 0,2$ & $1,0^{*} \pm 0,2$ & $0,5 \pm 0,1$ \\
\hline Peur & $4,1^{*} \pm 0,6$ & $3,4^{*} \pm 0,4$ & $2,3 \pm 0,5$ \\
\hline Santé générale & $3,4 \pm 0,2$ & $2,9^{*} \pm 0,3$ & $3,8 \pm 0,2$ \\
\hline $\begin{array}{l}\text { Pression artérielle systolique } \\
(\mathrm{mm} / \mathrm{Hg})\end{array}$ & $128^{* * *} \pm 1,7$ & $138^{* *} \pm 1.4$ & $114 \pm 1,1$ \\
\hline Acide vanyl mandélique & $8,1^{*} \pm 1,2$ & $9,5^{* *} \pm 1,6$ & $4,36 \pm 0,8$ \\
\hline
\end{tabular}

${ }^{*} \mathrm{p}<0,05, * * \mathrm{p}<0,01, * * * \mathrm{p}<0,001$ comparaison par rapport au groupe témoin / compared to the control group.

rentes sous-échelles du «Cornell Medical Index » sont significativement plus élevés pour les groupes d'Abadia et de Goiânia par rapport au groupe non exposé. Le score de détresse le plus élevé est observé pour le groupe d'Abadia. La perception du danger est significativement plus importante pour les groupes d'Abadia et de Goiânia 
par rapport au groupe non exposé. Le score de mesure de la peur est plus élevé dans le groupe d'Abadia que dans le groupe de Goiânia. Le groupe de Goiânia présente le plus mauvais score sur l'état de santé perçu. Pour les tests psychotechniques, le groupe d'Abadia est le plus affecté, puis celui de Goiânia, tous deux ayant des tests inférieurs à ceux des témoins. Les niveaux d'acide vanyl mandélique urinaire sont supérieurs dans les groupes de Goiânia et d'Abadia à ceux observés dans le groupe non exposé de même que la pression artérielle systolique. Ainsi, trois ans et demi après l'accident, un excès de symptomatologie liée au stress est observé chez les personnes irradiées et celles vivant près du site de stockage.

\section{L'accident de Tchernobyl}

Le 26 avril 1986, l'explosion du réacteur $n^{\circ} 4$ de la centrale nucléaire de Tchernobyl en Ukraine a entraîné des contaminations élevées en Ukraine, Biélorussie et Russie et, à un degré moindre, dans divers pays européens voisins. Plusieurs millions de personnes ont été impliquées à des degrés divers qu'il s'agisse des « liquidateurs » chargés de décontaminer et nettoyer le site autour du réacteur, des habitants évacués dans la semaine suivant l'accident et relogés dans des zones parfois non indemnes de contamination, ou encore, des résidants des zones contaminées (UNSCEAR, 1988). En 1995, on avait diagnostiqué dans les pays de la Communauté des États Indépendants (CEI) plus de 600 cas de cancer de la thyroïde chez les enfants âgés de moins de 15 ans (Bard et al., 1997). L'accident a eu des conséquences économiques et sociales lourdes qui ont touché à des degrés divers les 280 millions de personnes des trois pays de la CEI.

L'euphémisation de la situation accidentelle dans les informations officielles, en totale contradiction avec les informations non officielles et la prise en charge matérielle de l'accident, a contribué à un climat durable de doute et de méfiance vis-à-vis des autorités scientifiques, médicales et politiques (Havenaar, 1996a; HériardDubreuil, 1994; Rumyantseva et al., 1996). La stratégie d'euphémisation adoptée par les autoritês s'est beaucoup appuyée sur le concept de « radiophobie ». Cette nouvelle entité nosologique caractérisait les manifestations de craintes exprimées par la population à l'égard de la situation radiologique. Ces craintes étaient présentées comme relevant d'une névrose de type phobique puisque jugées comme irrationnelles et sans fondement. Ce concept a progressivement été abandonné avec les changements politiques et aussi parce que son manque de pertinence clinique est apparu de plus en plus évident dans les suites de l'accident. Il a été remplacé à partir de 1991 par le concept de stress qui rendait mieux compte des phénomènes observés (Girard et Hériard-Dubreuil, 1995). C'est à cette époque que la population est passée brutalement d'une situation de « sous » information à une situation de « sur » information sans être en mesure de distinguer ce qui résultait de l'accident de ce qui lui était attribué à tort (Tarabrina et al., 1996). 
La prise en charge des populations déplacées ou résidant dans les zones contaminées et les modalités de compensation des dommages ont certainement eu un effet aggravant, contribuant à freiner le retour à la vie normale, provoquant une ségrégation sociale et laissant peu d'initiatives aux individus (Hériard-Dubreuil, 1994). Parallèlement, une grave désintégration du tissu social et une grave crise économique se sont produites dans les pays de la CEI. Dans les dix dernières années, les indicateurs de santé des populations de la CEI se sont détériorés comme le montre l'accroissement de la mortalité infantile et la diminution de l'espérance de vie (Adveev et al., 1997).

Ainsi, les risques pour la santé posés par la contamination radioactive à long-terme et les nombreuses incertitudes et controverses qui les entourent, le déracinement des communautés dû à leur évacuation et au changement des modes de vie, caractérisent le contexte dans lequel les populations vivent actuellement.

\subsection{Effets sur la santé mentale}

Les études disponibles concernent d'une part, les travailleurs de la centrale, les intervenants des premiers jours et les liquidateurs et, d'autre part, la population générale en Ukraine, Biélorussie et Russie. Dans les pays hors CEI, les études publiées développent un point de vue psychosocial (opinions sur les risques, l'information, les autorités...) plutôt que les problèmes de santé mentale; aussi il n'en sera pas fait état dans cette synthèse.

\subsubsection{Travailleurs de la centrale, intervenants, liquidateurs}

Les études sont plus des relations cliniques approfondies de patients hospitalisés ou consultant, que des études épidémiologiques permettant d'évaluer l'impact de l'accident et de généraliser les résultats.

Chinkina (Chinkina, 1991) et Torubarov (Torubarov, 1991) ont suivi pendant quatre ans, 85 personnes atteintes du syndrome aigu d'irradiation. Les niveaux d'anxiété importants, présents dans les semaines qui ont suivi, étaient fonction de la gravité de l'atteinte somatique. Trois ans après, il existait une corrélation entre la gravité du syndrome aigu d'irradiation et la prévalence de névroses ou de tensions psychologiques $(80 \%$ pour le groupe de gravité sévère, $71 \%$ pour celui de gravité moyenne et $36 \%$ pour le groupe de faible gravité). Les troubles psychologiques persistants étaient généralement des sensations d'asthénie, de dévalorisation de soi, une labilité émotionnelle, l'anxiété et 1'hypochondrisme. La satisfaction professionnelle et les mesures de réhabilitation sociale se sont révélées être des facteurs protecteurs. 
Koscheyev a étudié la santé mentale d'une centaine de chefs opérateurs de la centrale de Tchernobyl entre juillet 86 et décembre 87 (Koscheyev et al., 1993). Les résultats ont été comparés à ceux d'un groupe de collègues d'une autre centrale testés en avril 1988. L'instrument de mesure utilisé était une traduction russe du Minnesota Multiphasic Personality Instrument (MMPI) (Graham, 1987). Les scores de symptômes de troubles psychiques étaient plus élevés pour le personnel de la centrale de Tchernobyl et la proportion de scores individuels considérés comme pathologiques était plus importante. Pour les questions explorant l'hypochondrie et la dépression, les scores ont augmenté au cours du temps. Bien que les sujets étudiés ne soient pas les mêmes aux différentes dates et que le groupe non exposé ait été étudié plus tardivement, les auteurs concluent à l'existence d'un niveau de stress supérieur pour le personnel de la centrale de Tchernobyl.

Afin de rechercher l'existence d'un état de stress post-traumatique parmi les personnes impliquées dans l'accident et ses suites, Tarabrina (Tarabrina et al., 1996), a réalisé l'examen clinique de 65 liquidateurs indemnes de pathologies somatiques dues aux rayonnements ionisants ou de pathologies cérébrales. Un état de stress post-traumatique a été diagnostiqué chez 21 liquidateurs (32\%) à l'aide du guide d'entretien «Structured Clinical Interview for DSMIIIR » (SCID) (Spitzer et al., 1989). Les 21 liquidateurs présentaient, pour les différentes échelles testées, des profils de réponse analogues à ceux observés sur d'autres populations souffrant d'un état de stress posttraumatique (comme les vétérans de la guerre du Vietnam), les scores restant toutefois plus faibles. Les liquidateurs avaient notamment leur attention fixée sur leur santé somatique. Un questionnaire portant sur le vécu de l'accident et la perception du risque lié aux rayonnements ionisants montrait le rôle déterminant de l'information et de l'appréciation personnelle dans le développement de ce syndrome.

\subsubsection{Population générale}

Il n'a pas été identifié d'étude épidémiologique en population générale dans l'année suivant l'accident. Dans les années suivantes, plusieurs études ont été faites sur les personnes vivant en territoire contaminé.

Dans le Projet International pour Tchernobyl (AIEA, 1991), les troubles du sommeil, les plaintes somatiques subjectives et le niveau de consommation d'alcool ont été étudiés au moyen d'un autoquestionnaire non standardisé pour des échantillons d'individus d'âges différents ( 4 ans, 12 ans, 20 ans, 40-50 ans, 60-70 ans) résidant dans des villages contaminés à plus de $600 \mathrm{kBq} / \mathrm{m}^{2}\left(15 \mathrm{Ci} / \mathrm{km}^{2}\right)$ et à moins de $37 \mathrm{kBq} / \mathrm{m}^{2}\left(1 \mathrm{Ci} / \mathrm{km}^{2}\right)$. Un niveau de «stress » élevé a été observé mais sans différer significativement selon le niveau de contamination des villages.

Une enquête d'opinion a été réalisée sur un échantillon de 3067 personnes sélectionnées selon la méthode des quotas en Biélorussie, Russie et Ukraine (Drottz-Sjöberg et al., 1994; Rumyantseva et al., 1996). Ces personnes résidaient dans des zones 
contaminées, des zones adjacentes non contaminées, des zones de relogement ou des zones éloignées non contaminées. Le niveau de détresse psychologique liée à l'accident, mesuré par un questionnaire ad hoc, était généralement élevé. Les personnes relogées ou vivant en zone contaminée présentaient un niveau de détresse plus élevé que les personnes des zones non exposées. Le meilleur prédicteur de détresse était la peur d'effets sur la santé dans le futur pour soi-même ou sa famille. Entre 25 et $50 \%$ des personnes interrogées disaient utiliser des médicaments sédatifs, le pourcentage le plus élevé étant observé parmi les relogés.

\section{TABLEAU V}

Scores moyens et prévalence de scores $>2$ au General Health Questionnaire 28 (d'après Viinamäki et al., 1995).

Mental wellbeing in study and control groups adjusted on age by sex. Prevalence of people with minor mental disorder by sex (GHQ28 score > 2) (Viinamäki et al., 1995).

\begin{tabular}{|c|c|c|c|c|}
\hline 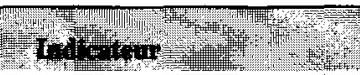 & 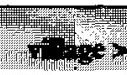 & 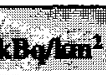 & (x) & 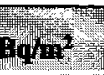 \\
\hline (1) & hompes & Apumps & Haphes & Trinines \\
\hline $\begin{array}{l}\text { score moyen } \\
\text { ajusté sur l'age }\end{array}$ & $2,2 *$ & $\mathbf{3 , 8 *}$ & 1,4 & 1,7 \\
\hline $\begin{array}{l}\text { prévalence d'un score } \\
\text { supérieur à } 2\end{array}$ & $26 \%$ & $48 \% *$ & $28 \%$ & $34 \%$ \\
\hline
\end{tabular}

* différence significative au risque $5 \%$ entre les deux villages. / Significant difference at $5 \%$ risk between the two villages.

Sept ans après l'accident, Viinamäki (Viinamäki et al., 1995) a conduit une étude transversale dans la région de Briansk en Russie sur 325 personnes de plus de 14 ans vivant en zone contaminée ( $\left.>1300 \mathrm{kBq} / \mathrm{m}^{2}\right)$ et 278 personnes en zone peu contaminée $\left(<37 \mathrm{kBq} / \mathrm{m}^{2}\right)$. Une version russe du General Health Questionnaire (GHQ28) servait d'indicateur de détresse psychologique, les caractéristiques sociodémographiques et économiques, la perception de sa capacité au travail et l'existence d'un soutien social étant étudiées comme facteurs aggravants ou protecteurs. Certains résultats sont présentés dans le tableau V. Le score global ajusté sur l'âge était significativement plus élevé pour les personnes du groupe exposé. Chez les femmes, qui présentaient des scores plus élevés au GHQ, l'âge, la détérioration du soutien social, la perception d'un mauvais état de santé et l'existence de difficultés économiques étaient des facteurs aggravants de détresse psychologique. Chez les hommes, les facteurs aggravants étaient l'âge et le mauvais état de santé perçu.

Dans l'étude de Havenaar (Havenaar et al., 1995, 1996a,b,c,d) réalisée sept ans après l'accident, un échantillon représentatif de la population d'âge compris entre 18 et 65 ans vivant dans une région hautement contaminée (Gomel, Biélorussie, 
1617 sujets) a été comparé à un échantillon provenant d'une région équivalente au plan socio-économique mais beaucoup moins contaminée (Tver, Russie, 1427 sujets). La détresse psychologique a été abordée au moyen de trois questionnaires validés au cours de l'étude dans la langue et le contexte culturel russes (Havenaar et al. 1996d) : le General Health Questionnaire GHQ12, le Bradford Somatic Inventory (Mumford et al., 1991) qui explore les expressions somatiques de la détresse psychologique et le Goldberg Scale for Anxiety and Disorders (Goldberg et al., 1988) qui détecte les symptômes d'anxiété. Des questions ont été ajoutées pour obtenir une information sociodémographique, pour évaluer la perception du sujet sur sa santé, connaître le nombre de visites chez le médecin et l'utilisation de médicaments prescrits durant le dernier mois. En fonction des scores obtenus au GHQ12, deux sous-échantillons ont été constitués, l'un de 265 personnes à Gomel et l'autre de 207 personnes à Tver. Ces personnes ont ensuite répondu aux trois questionnaires et ont fait l'objet d'un examen clinique somatique et psychiatrique standardisé. Les diagnostics cliniques somatiques étaient classés selon le Code International des Maladies (CIM9) (ICD 9, 1977). L'examen psychiatrique clinique reposait sur le Munich Diagnostic Check-list (Havenaar et al., 1995). Un volet de diagnostic des troubles somatoformes et un volet de diagnostic de l'état de stress post-traumatique ont été rajoutés pour les besoins de l'étude. Un modèle logistique multivarié permettait d'estimer les risques relatifs (standardisés sur le sexe, l'âge, le statut marital et le niveau d'éducation) entre Gomel et Tver et entre population générale et groupes présentant des caractéristiques pouvant être associées à des effets plus importants. Sur la base des résultats obtenus sur les deux souséchantillons, les prévalences de pathologies somatiques, de troubles anxieux ou de l'humeur et de signes de détresse psychologique étaient estimées pour la population générale. Les facteurs possiblement aggravants recherchés étaient les suivants : avoir été évacué, vivre dans une zone contaminée contrôlée, être mère d'un enfant de moins de 18 ans, et être liquidateur.

Les résultats sont présentés dans le tableau VI. Le fait le plus frappant est que plus de $50 \%$ d'une population active a priori en bonne santé perçoive son état de santé comme mauvais et présente des signes de détresse psychologique (score au GHQ12 > 2). Outre les scores au GHQ12, les prévalences de pathologies somatiques et de troubles psychiatriques sont beaucoup plus élevées que dans les pays occidentaux. Le pourcentage élevé d'individus qui ont eu recours aux soins confirme cette tendance.

Il n'y a pas de différence significative sur la prévalence des troubles cliniques somatiques ou psychiatriques entre Tver et Gomel. Par contre, les scores au GHQ sont significativement supérieurs à Gomel qui a été la région la plus contaminée. Au sein de cette région, il n'y a pas de différence entre population habitant en zone hautement contaminée et population générale. Les scores élevés au GHQ12 et les pathologies cliniques psychiatriques sont plus fréquentes chez les femmes et surtout chez les mères. Les scores sont aussi significativement plus élevés chez les évacués. 


\section{TABLEAU VI}

Indices cliniques et état de santé auto-déclarés à Gomel, Biélorussie, et à Tver, Russie (d'après Havenaar et al., 1996 a,b).

Self reported and clinical health indices in Gomel and Tver (Havenaar et al., 1996 a,b).

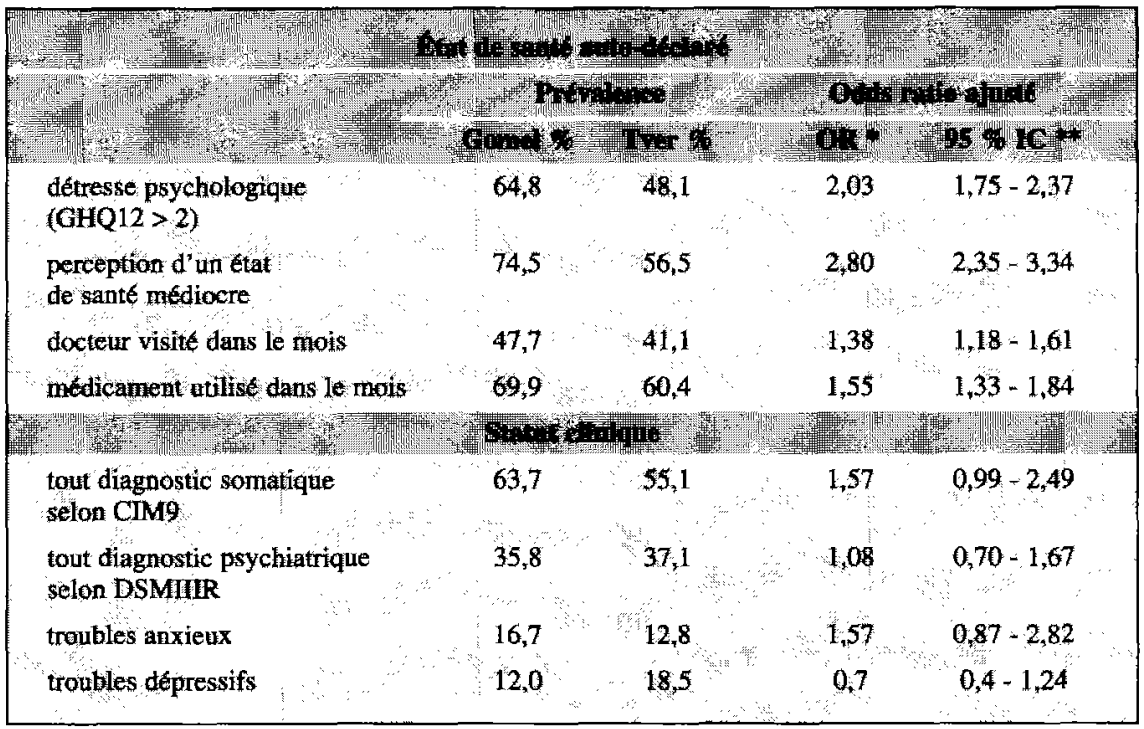

* OR : Odds Ratio ajusté sur le sexe, l'âge, le statut marital et l'éducation

$I$ odds ratio adjusted on age, sex, marital status and education.

** IC $95 \%$ : Intervalle de Confiance à $95 \%$

$/$ confidence interval at $95 \%$.

\subsubsection{Discussion des études sur Tchernobyl}

Selon l'OMS (WHO, 1995), les conséquences psychologiques constituent un des problèmes majeurs de santé publique de l'accident de Tchernobyl. Cependant, il existe peu d'études permettant d'évaluer l'impact de l'accident sur la santé mentale et cellesci ont été mises en place tardivement. L'appréciation de l'impact de Tchernobyl dans le court terme est donc très difficile.

Les publications disponibles présentent souvent des limitations méthodologiques importantes, particulièrement les études portant sur le personnel de la centrale, les patients ou les liquidateurs. Les modes de sélection des échantillons, l'absence de groupe comparatif adéquat et de description détaillée des critères diagnostiques rendent difficile l'appréciation de l'impact de l'accident sur ce type de population. Il semble cependant exister un excès de symptômes de pathologies sub-cliniques au sein du personnel de la centrale dans les deux années qui ont suivi l'accident et des états de stress post-traumatiques chez les liquidateurs. 
La situation est meilleure pour les études s'intéressant, plusieurs années après la survenue de l'accident, à la population générale des territoires touchés. La taille des échantillons sélectionnés est supérieure. Un effort a été fait pour obtenir des échantillons représentatifs des populations ciblées et des groupes non exposés. Enfin le recours aux instruments standardisés de diagnostic et d'évaluation des symptômes est fréquent.

Le résultat le plus constant est l'existence, plusieurs années après l'accident, d'une perception de l'état de santé physique et psychologique plus mauvaise au sein des régions exposées que dans les zones non exposées (Rumyantseva et al., 1996; Viinamäki et al., 1995; Havenaar et al., 1996b). Cette mauvaise perception ainsi que la prévalence des troubles psychologiques et psychiatriques sont globalement beaucoup plus importantes au sein de la CEI qu'en Occident. Il existe des raisons objectives à cela, comme le montrent les statistiques publiées par l'OMS sur la morbidité au sein des populations de la CEI (Havenaar et al., 1996b) et la constante diminution de l'espérance de vie depuis 15 ans dans les pays de l'exURSS (Adveev et al., 1997). En revanche, la prévalence de troubles psychiatriques cliniquement avérés ne semble pas différer entre les zones exposée et non exposée. La mise en place tardive des études, ainsi que la prévalence élevée des troubles psychiatriques peuvent avoir masqué des différences faibles.

\section{Discussion générale}

Les études de l'impact psychologique et psychiatrique des accidents de Three Mile Island, de Goiânia et de Tchernobyl ont montré, de façon remarquablement constante dans des contextes culturels et accidentels pourtant très différents, l'existence d'une augmentation significative et durable des scores de symptômes psychologiques au sein des populations exposées. Ce résultat est en faveur d'un impact réel sur le bien être psychique. Des manifestations neuro-endocriniennes et des signes somatiques objectifs (tension artérielle) observés dans certaines études après l'accident de Three Mile Island ou celui de Goiânia corroborent ce résultat et l'existence d'un état de stress chronique persistant au sein de ces populations. Néanmoins les manifestations observées restent la plupart du temps dans les limites de la «normalité ».

Divers auteurs ont qualifié les effets mesurés par les échelles de symptômes de « détresse psychologique » ou de « démoralisation » pour définir le sentiment de désespoir, de dévalorisation et de détérioration de son état de santé (Dohrenwend et al., 1981 ; Havenaar et al., 1996b). Cette détresse se distingue des troubles psychiatriques cliniques qui constituent une réponse pathologique.

La détresse psychologique n'est pas distribuée de façon homogène au sein des populations étudiées. Certaines caractéristiques sont associées à une détresse psychologique plus importante : l'âge, se trouver dans une situation personnelle où le tissu social et le statut économique se sont dégradés, avoir été évacué ou être mère 
d'un enfant de moins de 18 ans. Les mères expriment une nette inquiétude à l'égard de l'état de santé de leurs enfants. Des raisons objectives alimentent cette inquiétude, comme l'excès de cancers de la thyroïde observé après l'accident de Tchernobyl. Le fait d'avoir été évacué et relogé est aussi associé à une détresse psychologique plus élevée. Le déplacement forcé sans information associée, le passage d'un mode de vie rural à un mode de vie urbain, l'éclatement des familles, la discrimination de la part de la population hôte peuvent expliquer ce résultat (Hériard-Dubreuil, 1994).

L'existence de troubles mentaux clairement diagnostiqués et spécifiques après un accident radiologique n'a pas été, jusqu'à présent, suffisamment recherchée. Une seule étude a mis en évidence un excès de troubles anxieux et dépressifs dans une population exposée comparée à une population témoin. Il s'agit des mères d'enfants en bas âge résidant à proximité de la centrale de Three Mile Island (Bromet et al., 1982). Cet excès n'a été observé que dans la première année après l'accident. La plupart des études épidémiologiques sur les effets psychologiques et psychiatriques des accidents radiologiques ont été beaucoup plus tardives.

Selon Green, il faut s'attendre à ce que les syndromes d'état de stress post-traumatique soient moins intenses et moins fréquents dans les suites des accidents entraînant la libération d'un produit toxique et n'impliquant pas la perception directe et sensorielle du danger (Green et al., 1994). Une étude semble mettre en évidence l'existence de tels syndromes parmi les liquidateurs de Tchernobyl. De même, après l'accident de Three Mile Island, l'existence plus fréquente de pensées intrusives au sujet de l'accident a été mise en évidence dans un échantillon de la population générale exposée (Baum et al., 1993).

L'origine neurologique de manifestations psychiatriques ou de moins bons résultats aux tests psychométriques au sein des populations ne peut être exclue. Des atteintes neurologiques et des altérations des performances intellectuelles peuvent être provoquées par des expositions aux radiations, particulièrement chez les enfants dans les premières années (Ron et al., 1982) ou au moment de la gestation entre la $8^{\mathrm{e}}$ et la quinzième semaine (Dunn et al., 1990). Une association significative a été mise en évidence entre des irradiations par rayons $X$ de la tête (pour teigne) chez l'enfant et l'augmentation de symptômes psychiatriques (Albert et al., 1966). Ce résultat peut laisser penser que le mécanisme sous-jacent est celui de lésions neurologiques radioinduites. Concernant Tchernobyl, à notre connaissance il n'existe pas dans la littérature internationale d'étude épidémiologique sur les manifestations psychiatriques d'origine neurologique au sein des populations exposées.

\section{Conclusion}

La détresse psychologique chronique ne doit pas être sous-estimée. Elle traduit une atteinte réelle du bien-être général et de l'état de santé de la population et touche une part importante de celle-ci durant des périodes prolongées après la survenue de 
l'accident. Elle peut favoriser le développement de troubles psychiatriques et entrâ̂ner des changements de comportements à l'égard de la santé, soit en suscitant la recherche de soins, soit en favorisant des comportements à risque. Elle peut ainsi avoir un impact non négligeable en termes de santé publique. Elle peut enfin compliquer la prise en charge et la gestion des conséquences des accidents radiologiques sur les populations exposées.

Il existe un grand besoin de connaissance pour évaluer l'impact des accidents radiologiques majeurs sur la santé mentale et en prévenir ou atténuer les effets. Cette revue montre les difficultés méthodologiques faisant obstacle à une évaluation correcte d'un tel impact. À partir de l'expérience acquise, différentes voies de recherche et de réflexion peuvent être envisagées :

1) la construction d'outils pertinents de mesure de l'exposition, prenant notamment en compte les aspects de perception et de vécu de l'accident et leur grande variabilité individuelle;

2) la construction et la validation d'outils de mesure des effets psychologiques et psychiatriques mieux adaptés au contexte des accidents radiologiques en accord avec les phénomènes psychophysiologiques sous-jacents;

3) une meilleure identification et compréhension des déterminants et modificateurs de risque d'impact psychologique sur les individus et les groupes auxquels ils appartiennent;

4) une meilleure prise en compte des réactions psychologiques des populations confrontées aux expositions radiologiques accidentelles dans la planification des aides sociales, économiques, psychologiques et médicales, aussi bien immédiatement après l'accident que dans le long terme.

\section{RÉFÉRENCES}

AIEA (1989) L'accident radiologique de Goiania. Agence Internationale de l'Energie Atomique, Vienne, Autriche.

AIEA (1991) The International Chernobyl Project, An Overview, Assessment of Radiological Consequences and Evaluation of Protective Measures, Report by an International Advisory Committee. Vienna, Austria.

Adveev A., Blum A., Zakharov S., Andreev E. (1997) Réaction d'une population hétérogène à une perturbation. Un modèle d'interprétation des évolutions de mortalité en Russie. Population, 1, 7-44.

Albert R.E., Omran A.R., Brauer E.W., Dove D.C., Cohen N.C., Schmidt H., Bamuring R., Morrill S., Schulz R., Baer R.L. (1966) Follow up study of patients treated by X-ray for tinea capitis. American J. Public Health, 56, 2114-2120. 
Ph. PIRARD et al.

Bard D., Verger P., Hubert P. (1997) Chernobyl ten years after: health consequences. Epidemiologic Reviews, 19, 187-204.

Bartlett G.S., Houts P.S., Bymes L.K., Miller R.W. (1983) The Near Disaster at Three Mile Island. Int. J. Mass Emerg. Disasters, 1(1), 19-43.

Baum A., Cohen L. (1993) Hall M. Control and Intrusive Memories as Possible Determinants of Chronic Stress. Psychosom. Med., 55, 274-286.

Brodman K., Erdman A.J., Wolff H.G. (1974) Cornell Medical Index, Cornell University Medical College press, New York.

Bromet E.J., Parkinson D.K., Schulberg H.C., Dunn L. O, Condek P.C. (1982) Mental Health of Residents near the Three Mile Island Reactor: a Comparative Study of Selected Groups. J. Prev. Psychiatr., $1,25-276$.

Bromet E.J., Parkinson D.K., Dunn L.O. (1990) Long Term Mental Health Consequences of the Accident at Three Mile Island. Int. J. Ment. Health, 19, 48-60.

Chinkina O.V. (1991) Psychological Characteristics of Patients Exposed to Accidental Irradiation at the Chernobyl Atomic-Power Station. in The Medical Basis for Radiation-Accident Preparedness, III The psychological perspective. (Ricks R.C., Berger M.E., O'Hara F.M., eds.) pp. 93-103. N.Y. Elsevier Science Publishing, New-York.

Collins D.L., de Carvalho A.B. (1993) Chronic Stress from the Goïania Cs-137. Radiation Accident. Behav. Med., 18, 149-157.

Cornely P., Bromet E.J. (1986) Prevalence of Behavior Problems in Three-Years Old Children Living near Three Mile Island: a Comparative Analysis. J. Child. Psychol. Psychiatr, 27, 489-498.

Derogatis L. (1977) The SCL-90 Manual I: Scoring, Administration and Procedures for the SCL-90. Baltimore: The John Hopkins University School of Medicine, Clinical Psychometric Units.

Dew M.A., Bromet E.J., Schulberg H.C., Dunn L.O., Parkinson D.K. (1987) Mental Health Effects of the Three Mile Island Nuclear Reactor Restart. Am. J. Psychiatr, 144, 1074-1077.

Dew M.A., Bromet E.J. (1993) Predictors of Temporal Patterns of Psychiatric Distress During 10 Years Following the Nuclear Accident at Three Mile Island. Soc. Psychiatr. Psychiatr. Epidemiol., $28,49-55$.

Dohrenwend B.P., Dohrenwend B.S., Wareiht G.J., Bartlett G.S., Goldsteen R.L., Goldsteen K., Martin J.L. (1981) Stress in the Community: a Report to the President's Commission on the Accident at Three Mile Island. Ann. N.Y. Acad. Sci., 365, 159-175.

Dohrenwend B.P. (1983) Psychological implications of nuclear Accidents: the case of Three Mile Island. Bull. N.Y. Acad. Med., 59, 1060-1076.

Drottz-Sjöberg B. M., Rumyantseva G.M., Arkhangelskaya H.V. et al (1994) Public reactions to the Chernobyl accident. Report from a data collection in 1993 in Russia, Belarus and Ukraine: social and psychological factors. Stockholm Center for Risk Research, Stokholm School of Economics, Stockholm, Sweden.

DSM-IIIR (1987) Diagnostic Statistical Manual IIIrd edition revised. Washington D.C., The American Psychiatric Association.

Dunn K., Yoshimaru H., Otake M., Annegers J.F., Schull W.J. (1990) Prenatal exposure to ionizing radiation and subsequent development of seizures. Am. J. Epidemiol., 131, 114-123.

Endicott J., Spitzer R. A (1978) Diagnostic Interview: the Schedule for Affective Disorders and Schizophrenia. Arch. Gen. Psychiatr, 35, 837-844. 


\section{CONSÉQUENCES DES ACCIDENTS RADIOLOGIQUES SUR LA SANTÉ MENTALE}

Fabrikant J.I. (1981) Health Effects of the Nuclear Accident at Three Mile Island. Health Phys., 40, $151-161$.

Fabrikant J.L. (1983) The Effects of the Accident at Three Mile Island on the Mental Health and Behavioural Responses of the General Population and Nuclear Workers. Health Phys., 45, 579-586.

Fleming R., Baum A., Gisriel M., Gatchel R.J. (1982) Mediating Influence of Social Support on Stress at Three Mile Island. J. Human Stress, september, 14-22.

Gatchel R.J., Schaeffer M.A., Baum A. (1985) A Psychophysiological Field Study of Stress at Three Mile Island. Psychophysiology, 22, 175-181.

Girard P., Hériard-Dubreuil G. (1995) La dimension du stress dans la gestion accidentelle et post-accidentelle de Tchernobyl. Conférence internationale « Radioprotection et Médecine » Montpellier, $28-30$ juin 1995.

Goldberg D.P. (1978) Manual of the General Health Questionnaire (National Foundation for Educationnal Research Eds.), Windsor.

Goldberg D., Bridges K., Duncan-Jones P., Grayson D. (1988) Detecting Anxiety and Depression in General Medical Settings. BMJ, 297, 897-9.

Graham J.R. (1987) The MMPI. A Practical Guide (second edition). Oxford University Press, New York.

Green B.L., Lindy J.D., Grace M.C. (1994) Psychological Effects of Toxic Contamination. In: Individual and Community Responses to Trauma and Disaster. (Ursano R.J., McCaughey B.G., Fullerton B.R. Eds). Cambridge University Press.

Havenaar J.M., Rumyantseva G.M., Filipenko V.V., van den Brink W., Poelijoe N.W., van den Bout J., Romasenko L. (1995) Experiences with a Checklist for DSMIIIR in the Russian Federation and Belarus. A Study about the Inter-Rater Reliability and the Concurrent Validity of the Munich Diagnostic Checklist for DSMIIIR. Acta Psychiatr. Scand., 92, 419-424.

Havenaar J.M. (1996a) After Chernobyl-Psychological Factors Affecting Health after a Nuclear Disaster. Thèse soutenue le 23 avril 1996 CIP-Gegevens Koninklijke Bibliotheek, Den Haag 1996; 175 p.

Havenaar J.M., Savelkoul T.J.F., van den Bout J., Bootsma P.A. (1996b) Psychological Consequences of the Chernobyl Disaster. A Survey of Psychological and Physical Well-Being in an Exposed and a Non-Exposed Population Sample. In: The radiological consequences of the Chernobyl accident (Karaoglou A., Desmet G., Kelly G.N., Menzel H.G., eds.) pp. 435-442. European Commission, Minsk, Belanus.

Havenaar J.M., van den Brink W., van den Bout J., Kasyanenko A.P., Poelijoe N.W., Wohlfarth T., MeijlerIljina L.I. (1996c) Mental Health Problems in the Gomel Region (Belarus). An Analysis of Risk Factors in an Area Affected by the Chernobyl Disaster. Psychol. Med., 26, 845-855.

Havenaar J.M., Poelijoe N.W., Kasyanenko A.P., van den Bout J., Koeter M.W.J. (1996d) Screening for Mental Health Problems in an Area Affected by the Chernobyl Disaster. The Reliability and Validity of Three Psychiatric Screening Questionnaires in Belarus. Psychol. Med., 26, 837-844.

Hériard-Dubreuil G. (1994) Premier bilan des effets psychiques et sociaux de l'accident de Tchernobyl. Radioprotection, 29, 363-376.

Hiller W., Zaudig M., Mombour W. (1990) Development of Diagnostic Checklists for Use in Routine Clinical Care. A guideline Designed to Assess DSMII-R Diagnoses. Arch. Gen. Psychiatr., 47, 782-784.

Horowitz M., Wilner N., Alvarez W. (1979) Impact of Event Scale: Measure of Subjective Stress. Psychosom. Med. 41, 209-218.

Houts P.S., Hu T.W., Henderson R.A., Cleary P.D., Tokuhata G. (1984) Utilisation of Medical Care Following the Three Mile Island Crisis. Am. J. Public Health, 74, 140-142. 
ICD 9 (1977) International Statistical Classification of Diseases and Related Health Problems 9th Revision. World Health Organisation Geneva.

ICD 10 (1993) Intemational Statistical Classification of Diseases and Related Health Problems IOth Revision. World Health Organisation Geneva.

Kemeny J.G., Babbitt B., Haggerty P.E. et al. (1979) Report of the President's Commission on the Accident at Three Mile Island: the Need for Change - The Legacy of TMI. Washington D.C. ISBN 0 93578-003.

Koscheyev V.S., Marteus V.K., Kosekov A.A., Lartzev M.A., Leon G.R. (1993) Psychological Status of Chernobyl Nuclear Power Plant Operators after the Nuclear Disaster. Brief Report. J. Trauma. Stress, 6, 561-568.

Kovess V. (1986) Epidémiologie des maladies mentales dans Encyclopédie Médico-Chirurgicale, psychiatrie 1-37878A10:2p.

Mc Leod G.K. (1982) A Role for Public Health in the Nuclear Age. Am. J. Public Health, 72, $237-238$.

Mileti D.S., Hartsough D.M., Madson P., Hufnagel R. (1984) The Three Mile Island Incident: a Study of Behavioral Indicators of Human Stress. Int. J. Mass Emerg. Disasters, 2, 89-113.

Mumford D.B., Bavington J.T., Bhatnagar K.S., Hussain Y., Mirza S., Naraghi M.M. (1991) The Bradford Somatic Inventory. A Multi-Ethnic Inventory of Somatic Symptoms Reported by Anxious and Depressed Patients in Britain and the Indo-Pakistan Subcontinent. B. J. Psychiatr., 158, 379-386.

Petterson J.S. (1988) Perception vs. Reality of Radiological Impact: the Goiania Model. Nucl. News, 31, 84-90.

Richman N., Graham P.J. (1971) A Behavioural Screening Questionnaire for Use with 3 Year Old Children. Preliminary Findings. J. Child. Psychol. Psychiatry, 12, 5-33.

Ron E., Modan B., Floro S. Harkedar I., Gurewitz R. (1982) Mental function following scalp irradiation during childhood. Am. J. Epidemiol., 116, 149-160.

Rumyantseva G.M., Drottz-Sjöberg B.M., Allen P.T., Arkhangelskaya H.V., Nyagu A.I., Ageeva L.A., Prilipko V. (1996) The Influence of Social and Psychological Factors in the Management of Contaminated Territories. In: The Radiological Consequences of the Chermobyl Accident (Karaoglou A., Desmet G., Kelly G.N., Menzel H.G., Eds.) pp. 443-452. European Commission, Minsk, Belarus.

Spitzer R.L., Williams J.B.W., Gibbon M., First M.D. (1989) Structured Clinical Interview for DSM-IIIR, American Psychiatric Press, Washington.

Tarabrina N., Lazebnaya E., Zelenova M., Lasko N. (1996) Chernobyl Clean-up Workers' Perception of Radiation Threat. Rad. Prot. Dosim., 68, 251-255.

Torubarov F.S. (1991) Psychological Consequences of the Chemobyl Accident from the Radiation Neurology Point of View. In: The Medical Basis for Radiation-Accident Preparedness, III The psychological perspective. (Ricks R.C., Berger M.E., O’Hara F.M., Eds.) pp. 81-91. N.Y. Elsevier Science Publishing, New-York.

UNSCEAR (1988) Sources, Effects and Risks of Ionising Radiation. 1988 Report to the General Assembly, with Annexes. United Nations Scientific Committee on the Effects of Atomic Radiation. United Nations, New York.

Viinamäki H., Kumpusalo E., Myllykangas M., Salomaa S., Kumpusalo L., Kolmakov S., Ilchenko I., Zhukovsky G., Nissinen A. (1995) The Chernobyl Accident and Mental Wellbeing - a Population Study. Acta Psychiatr. Scand., 91, 396-401.

WHO (1995) Health Consequences of the Chernobyl Accident. Results of the IPHECA Pilot Projects and Related National Programs. Summary Report. Geneva, Switzerland: World Health Organisation. 